\title{
Unilateral administration of a drug into the lung of a small animal
}

\author{
Soon Ho Cheong ${ }^{1,3}$, Young Il Yang ${ }^{2,3}$, Jie Yeon Seo ${ }^{3}$, Dong Hwa Jun ${ }^{1}$, Myoung Jin $\mathrm{Ko}^{1}$, Kwang Rae Cho ${ }^{1}$, \\ Sang Eun Lee ${ }^{1}$, Young Hwan Kim ${ }^{1}$, Se Hun Lim ${ }^{1}$, Jeong Han Lee ${ }^{1}$, and Kun Moo Lee \\ Departments of ${ }^{1}$ Anesthesiology and Pain Medicine, ${ }^{2}$ Pathology, Paik Hospital, Inje University , ${ }^{3}$ Paik Institute for Clinical Research, \\ Inje University, Busan, Korea
}

Background: The selective unilateral administration of drugs into a single lung of a rat is difficult because of the small airway diameter. Therefore, a simple method for unilateral administration into rat lung is needed.

Methods: Rats were assigned to 1 of 2 groups according to the direction of the catheter used for drug administration. Anesthetized rats were intubated, and curved epidural catheters were rotated up to a maximum of $90^{\circ}$ toward the left lung (group L) or right lung (group R). Bronchial catheters were then inserted via a tracheal tube and fixed. Methylene blue $(0.3 \mathrm{ml})$ was injected via the epidural catheter. Additionally, to compare survival rates, rats were assigned to one of two groups according to the drug administration route. In group T, bleomycin hydrochloride (20 $\mathrm{mg} / \mathrm{kg}$ ) in $0.3 \mathrm{ml}$ of phosphate-buffered saline (PBS) was administrated into the lung intratracheally via a tracheal tube. In group B, the same dose of bleomycin was administrated into the lung intrabronchially via a bronchial catheter, targeting the left lung.

Results: Gross examination revealed that targeted administration was $100 \%$ successful. Methylene blue was observed in the right lung of all rats in the R group and in the left lung of all rats in the $\mathrm{L}$ group. The survival rate was higher in group $\mathrm{B}$ than in group $\mathrm{T}$.

Conclusions: The intrabronchial method offers an advantage over tracheal administration as it decreases mortality and allows the administration of a drug unilaterally into a single lung or into a localized area without the need for double-lumen tubes or more invasive procedures. (Korean J Anesthesiol 2010; 58: 283-289)

Key Words: Bronchial, Rat, Unilateral.

\footnotetext{
Received: September 8, 2009. Revised: 1st, October 30, 2009; 2nd, November 3, 2009. Accepted: November 23, 2009.

Corresponding author: Soon Ho Cheong, M.D., Department of Anesthesiology and Pain Medicine, Paik Hospital, Inje University, 633-165, Gaegeum-dong, Jin-gu, Busan 614-735, Korea . Tel: 82-51-890-6520, Fax: 82-51-891-1455, E-mail: anesjsh@medimail.co.kr

This work was supported by Grant from Inje University, 2008.

(c) This is an open-access article distributed under the terms of the Creative Commons Attribution Non-Commercial License (http:// creativecommons.org/licenses/by-nc/3.0/), which permits unrestricted non-commercial use, distribution, and reproduction in any medium, provided the original work is properly cited.
} 


\section{Introduction}

Many studies on lung injury and the effects of drugs on the respiratory system in rats have relied upon tracheal drug administration $[1,2]$. In most lung research studies, a separate control subject is compared against the experimental animal. Alternatively, a procedure to administer drugs in a localized fashion into one lung would allow researchers to use the opposite lung of the same animal as a control, decreasing interindividual variation and systemic effects of the administered drug. To date, this type of approach has been used in large animals such as dogs or sheep through the use of endobronchial double-lumen tubes (DLTs) [3], endobronchial blockers, and Univent bronchial-blocker tubes (UBTs) [4]. However, no such products exist for use with small animals, as the limited airway diameter of small animals may make fabrication of DLTs difficult. Therefore, we developed a bronchial catheterization method for unilateral drug administration into rat lungs.

\section{Materials and Methods}

\section{Equipment and animal preparations}

A 16-gauge angiocath (45 $\mathrm{mm}$ in length, 20-mm hub, 1.77mm inner diameter; BD Angiocath Plus, Korea) was used as a tracheal tube and 20-gauge epidural nylon catheter (BD Perisafe, BD Medical System, Belgium) was used as a bronchial catheter (Fig. 1A). The Institutional Animal Care and Use Committee serving our University pre-approved all experimental procedures used in these studies. A total of 46 Sprague-Dawley rats $(250-350 \mathrm{~g})$ were selected as the model small animal. The rats were sedated with $5 \%$ enflurane- $\mathrm{O}_{2}$ ( $1 \mathrm{~L} / \mathrm{min})$ until the standing neutral position and eyelid closure reflexes were lost. After loss of the reflex response to tail clamping, endotracheal intubation was performed using the blind intubation technique with a 16-gauge angiocath, as described by Stark et al. [5]. The rats were maintained on spontaneous ventilation with a $3-5 \%$ enflurane inhalation during the procedure. The catheter was cut at a length greater than the distance from nose to abdomen of the rat and an appropriately curved 20-guage epidural nylon catheter was used for bronchial catheterization.

\section{Preliminary study}

Six rats were used to determine the appropriate depth of catheter insertion using X-ray and to confirm that the dye was administered unilaterally into a lung.

\section{Selective administration into unilateral lung}

The rats were divided into four groups ( $\mathrm{n}=10$ in each group); two groups according to the direction of the catheter for drug administration (group $\mathrm{R}$ and $\mathrm{L}$ ), and two groups according to the drug administration route (group $\mathrm{T}$ and $\mathrm{B}$ ).

In group $\mathrm{R}(\mathrm{n}=10)$, the catheter was rotated up to a maximum of $90^{\circ}$ to target the delivery of the drug to the right lung, and in group $\mathrm{L}(\mathrm{n}=10)$, the catheter was rotated up to a maximum of $90^{\circ}$ to target the left lung. In the L group, the bronchial catheter was rotated to the left prior to insertion via the tracheal tube (Fig. 1Ba). A similar procedure was used in group R (Fig. 1Bb, 1C). To minimize airway trauma during catheterization, the catheter was advanced slowly and carefully. Slight resistance was encountered as the catheter was advanced into the proximal airway of the lung, which was likely due to contact with the main stem or lobar bronchus. The catheters were then fixed, the rat was supine, and the cephalad end was raised to approximately a $45^{\circ}$ angle. Methylene blue $(0.3 \mathrm{ml})$ was then injected slowly for $5 \mathrm{~s}$ via the bronchial catheter using a 1-ml syringe. After the removal of the catheter and discontinuance of the enflurane
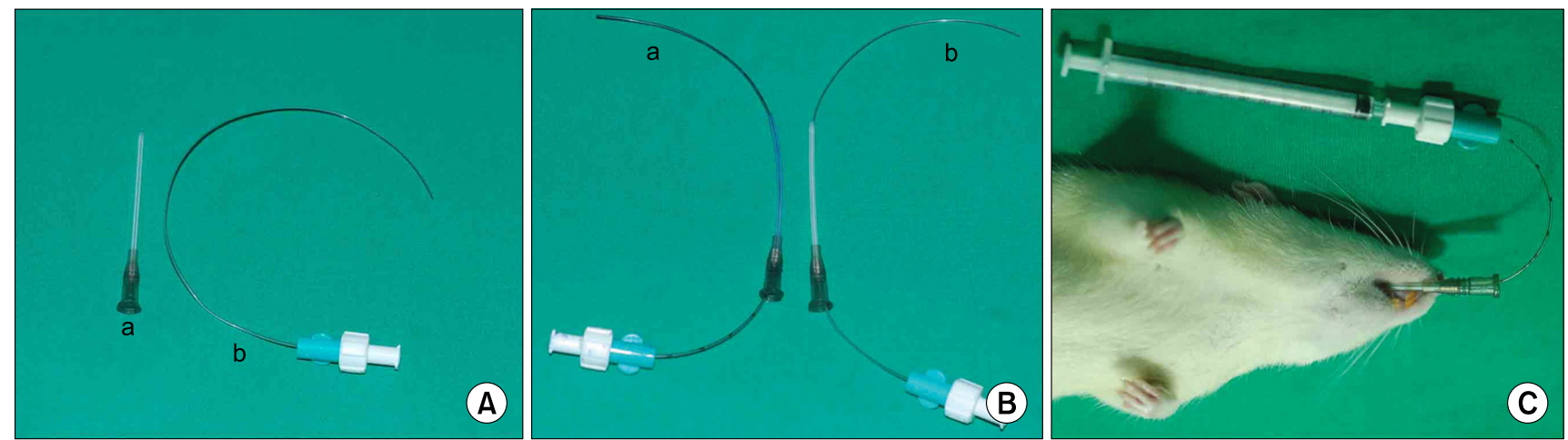

Fig. 1. Equipment used for endotracheal intubation and endobronchial catheterization, including the angiocath (Aa) and epidural catheter (Ab). The catheter was turned $90^{\circ}$ to the left $(\mathrm{Ba})$ or right $(\mathrm{Bb})$ for a selective catheterization into the left or right lung, respectively. Methylene blue was administered into the right lung after turning the catheter toward the right, followed by insertion and placement (C). 
inhalation, the head-up tilted position was maintained for 30 min to prevent methylene blue spillage through the carina. An i.p. injection of ketamine $(50-100 \mathrm{mg} / \mathrm{kg}$ ) was administered to supplement the inhalation anesthesia. The rats were then allowed to recover from the anesthesia. Upon showing active movement, the tracheal tube was removed, and the rats were taken to the animal care facility. The rats were sacrificed $1 \mathrm{~h}$ after methylene blue administration for gross review. Success of the experiment was judged by the presence of methylene blue in the correct lung upon this gross examination.

\section{Comparison of survival rate}

The rats were divided into two groups according to the drug administration route. In group $\mathrm{T}(\mathrm{n}=10)$, bleomycin hydrochloride $(20 \mathrm{mg} / \mathrm{kg})$ in $0.3 \mathrm{ml}$ of phosphate-buffered saline (PBS) was administrated into the lung intratracheally via the tracheal tube. In group B $(n=10)$, the same dose of bleomycin in $0.3 \mathrm{ml}$ of PBS was administrated into the lung intrabronchially via the bronchial catheter to target the left lung.

\section{Histology}

In group B rats, both lungs were harvested for microscopic analysis 28 days after the administration of bleomycin. Lung tissue was fixed by infusion with a $4 \%$ buffered formalin solution for $24 \mathrm{~h}$, embedded in paraffin, cut into serial sections, and stained with hematoxylin and eosin.

\section{Statistical analysis}

The success rate of the targeted lung administration and the survival rate were analyzed using the chi-square test. P values < 0.05 were considered statistically significant.
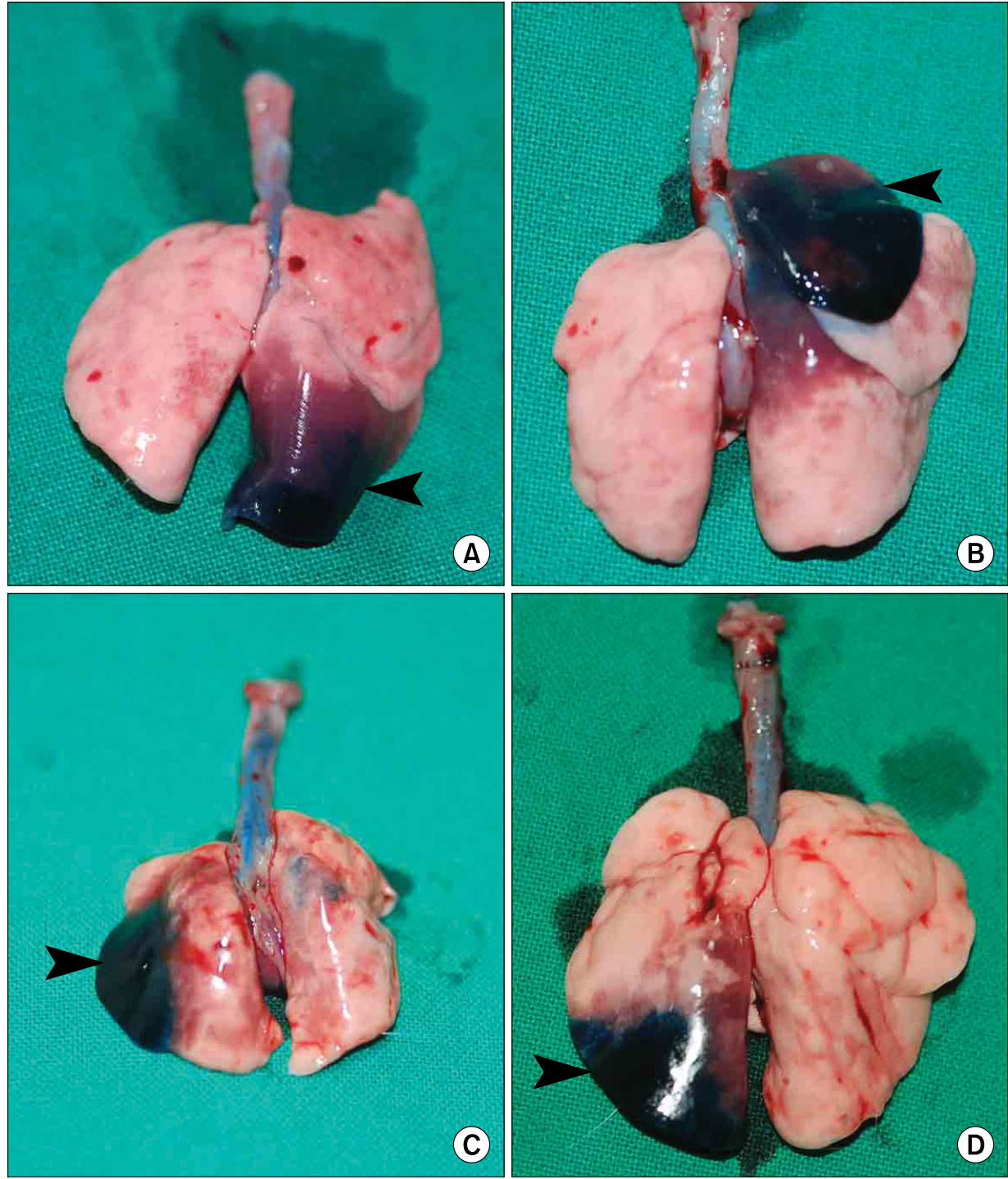

Fig. 2. Gross findings after the administration of methylene blue into a selective or localized area of the lung. The catheter was rotated $90^{\circ}$ to the right to target delivery of the drug into the right lung (A, B). Methylene blue $(0.3 \mathrm{ml})$ was dispersed into the lower part of the right caudal lung (arrow in A) and right cranial lung (arrow in B). The catheter was rotated $90^{\circ}$ to the left to target delivery of the drug into the left lung $(C, D)$. Methylene blue $(0.3 \mathrm{ml})$ was dispersed into the lateral (arrow in C) and lower (arrow in D) parts of the left lung. The arrows indicate methylene blue, and pulmonary edema is indicated by the light pink color surrounding the methylene blue stain. 


\section{Results}

The depth of the bronchial catheter from the upper incisor after insertion into the lung was $100 \pm 3 \mathrm{~mm}$ in the L group and $100 \pm 4 \mathrm{~mm}$ in the $\mathrm{R}$ group. The success rate of methylene blue administration into the target lung was $100 \%$ in both groups. In the $\mathrm{R}$ group, methylene blue was dispersed in the caudal lobe of nine rats and into the cranial lobe of the right lung in one rat (Fig. 2A and B, respectively). In the L group, methylene blue was dispersed into the peripheral (Fig. 2C) or lower part (Fig. 2D) of the left lung. Seven rats in group $\mathrm{T}$ died immediately after the administration of $0.3 \mathrm{ml}$ of bleomycin. In contrast, all rats in group B survived until their lungs were harvested 28 days after drug administration. Gross lesions were found in the left lungs of rats belonging to this group (arrow in Fig. 3B). The alveolar septa were infiltrated by mononuclear inflammatory cells and neutrophils and had widened due to the accumulation of fibrous tissue in left lung (Fig. 3D). However, no gross or microscopic lesions were observed in the right lungs of group B rats (Fig. 3C).

\section{Discussion}

Almendros et al. [6] performed differential lung ventilation
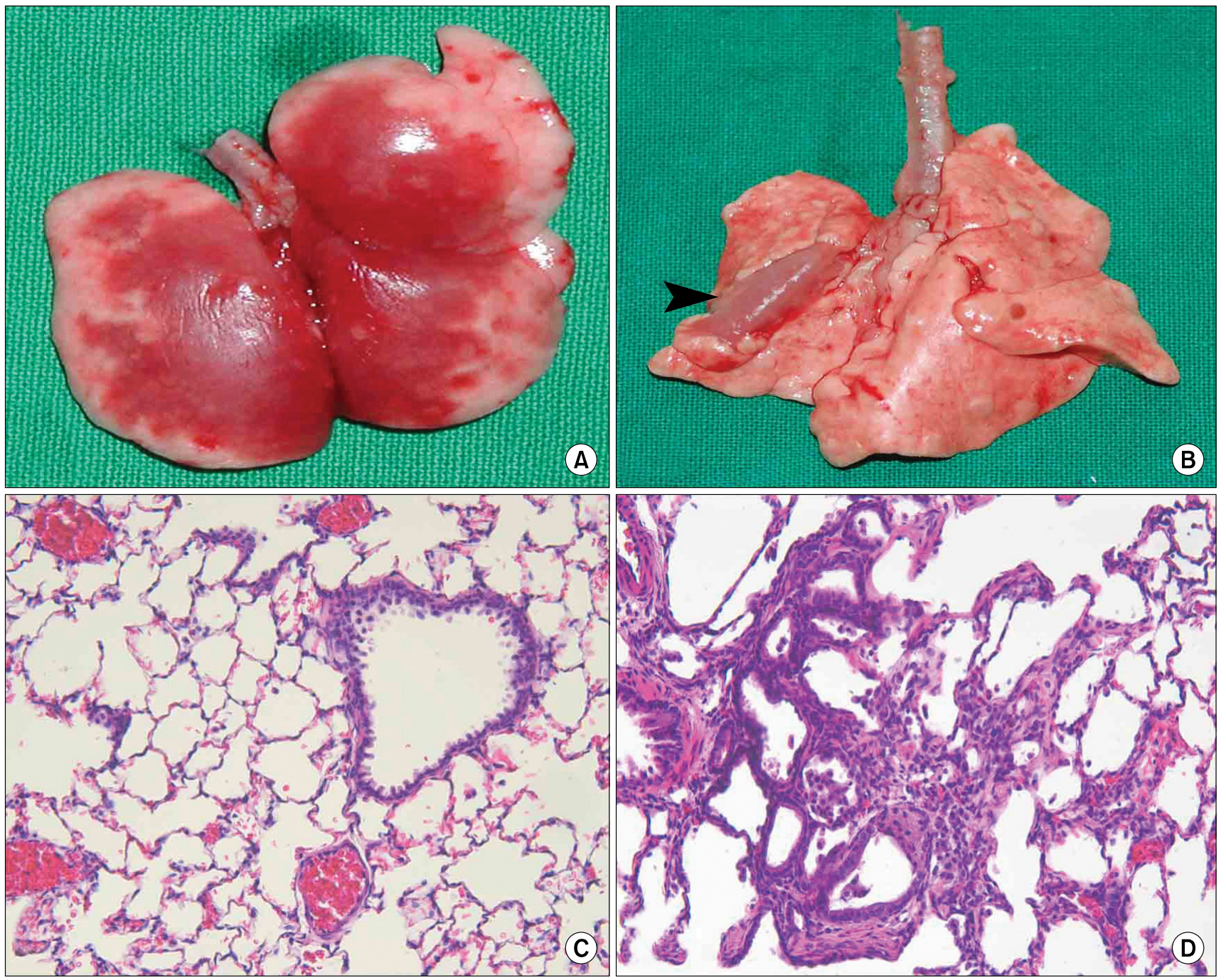

Fig. 3. Effects of the injection methods. Gross findings after the intratracheal (A) versus intrabronchial (B) administration of $0.3 \mathrm{ml}$ of bleomycin $(20 \mathrm{mg} / \mathrm{kg})$ into the lungs. Seven rats developed suspected pulmonary edema (reddish color in A) and died immediately after the intratracheal administration of bleomycin. However, rats that underwent intrabronchial administration of the same dose of bleomycin survived until their lungs were harvested 28 days later. Their lungs showed localized gross lesions (arrow in B). Microscopic findings 28 days after the intrabronchial administration of bleomycin into the left lung are shown in (C, D). No lesions were found in the right lung (C). Bleomycin-induced injury in the left lung caused multifocal interstitial pneumonitis and peribronchial fibrosis. The alveolar septa were infiltrated by mononuclear inflammatory cells and neutrophils and were enlarged due to the accumulation of fibrous tissue. (D) Magnification is $\times 200$. 
after a tracheotomy in a rat. In this previous study, the authors inserted two 16-gauge cannulae into each lung and sacrificed the animal after the procedure without recovery from anesthesia. Their method is difficult to use in research, which required recovery after the procedure, due to its extreme invasiveness. Clinically, the separation of both lungs is performed for various purposes, including one-lung ventilation, bronchoalveolar lavage (BAL), and surgery. DLTs, endobronchial blockers, and UBTs are employed for lung separation $[7,8]$. Our method of bronchial catheterization is similar to that of the UBT technique [9]. In one-lung ventilation techniques using UBTs, endotracheal intubation is performed and the head of the subject is turned to the right or left to target the respective lung following the advance of the bronchial blocker. In our study, the rat's head was not turned; instead, the curved bronchial catheter was rotated to the right or left, followed by insertion via the tracheal tube. In a clinical study, the blind rotation method was used in 32 of 44 cases for placement of the Univent blocker; the success rate of targeting the correct side was higher for the right (91.3\%) side, compared to the left (66.7\%) [10]. In our study, the success rate for targeting the correct lung was $100 \%$. The success rate would likely have been lower if the number of subjects were increased. The most important factor affecting the success rate in our study is assumed to be the degree of curvature of the catheter. Insertion into the correct bronchus is determined by the direction and lateral drive of the tip.

Rat lungs consist of five lobes: the 1) left lobe, 2) right caudal lobe, 3) right cranial lobe, 4) right middle lobe, and 5) accessory lobe [11]. The distance from the upper incisor to the carina in the rat used in this study was $76.1 \mathrm{~mm}$, found in an X-ray image. Dubaybo et al. [12] described a similar technique to that used in this study for the unilateral instillation of agents into rat lungs. In their study, the curvature of the bronchial catheter, insertion of the bronchial catheter through endotracheal tube with the curve concave toward the targeted direction, and the cephalad end in a raised position were similar to our technique. In their study, however, the targeted lung was only the right one, and they described the main-stem bronchus on the right side as being wider and more directly in line with the trachea than that on the left, ensuring entry of the tip of the tubing into the right lung. They emphasized that the length of the bronchial catheter and the volume and rate of instillation of the fluid were critically important. In our study, the targeted lung was the left (group L) in addition to the right lung (group R), and we performed other experiments for comparing the survival rate using bleomycin. Additionally, our study resulted in a success rate that was the same in either direction for a particular target lung, and the technique for unilateral instillation into the lung can decrease mortality that may be associated with bilateral lung injury.

In our preliminary study, methylene blue showed spillage into various areas of the lung. (Fig. 4A and B and data not shown). When the table was not elevated, methylene blue was found in the opposite lung after administration in the supine position (Fig. 4C, right arrow), and pneumothorax occurred due to careless insertion. Methylene blue found in the opposite lung was assumed to be due to spillage through the carina. This could have occurred as the methylene blue flowed back into the trachea and thus subsequent ventilation-induced flow into the opposite lung. Alternatively, an excess volume of the
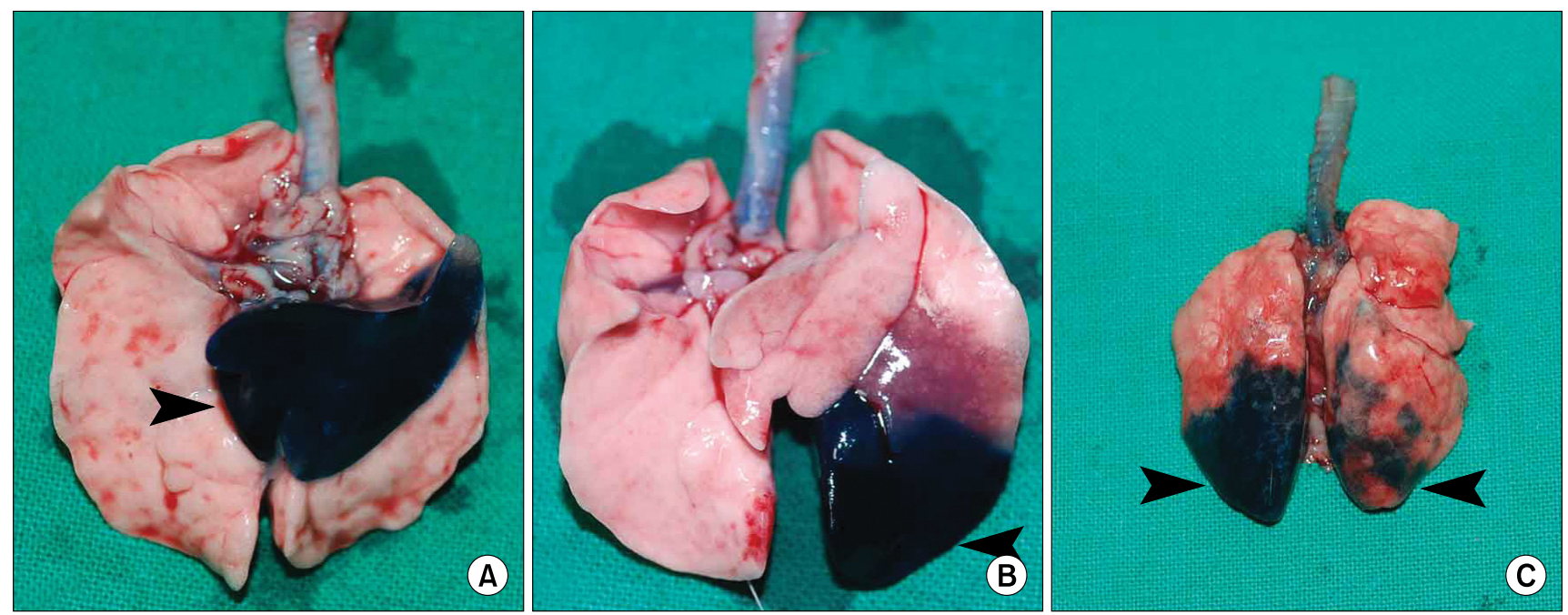

Fig. 4. Gross findings after administration of methylene blue using a straight catheter (A and B). Methylene blue was found in the accessory lobe (arrow in A) and left (arrow in B) lung. (C) Although the left lung was targeted in the supine position, methylene blue was observed in both lungs after administration (arrows in C). It is assumed that methylene blue administered into the left lung flowed over the carina into the right lung. 
administered substance could cause spillage into the opposite lung. In our study, we kept the raised head at a $45^{\circ}$ angle for 30 min under anesthesia with spontaneous ventilation after the administration of methylene blue, which prevented spillage over the carina. The duration that this raised position must be maintained to prevent spillage likely depends on the volume of the administered compound and the reaction of the compound with the lung tissue. Additionally, the appropriate volume likely depends on the age or weight of the animal. In a preliminary study using X-ray visualization with a contrast medium, we found that a relatively large volume $(0.5 \mathrm{ml})$ of fluid spilled into the opposite lung (Fig. 5G).

In our study, the tissue areas stained with methylene blue showed pulmonary edema-like lesions in most of the animals.
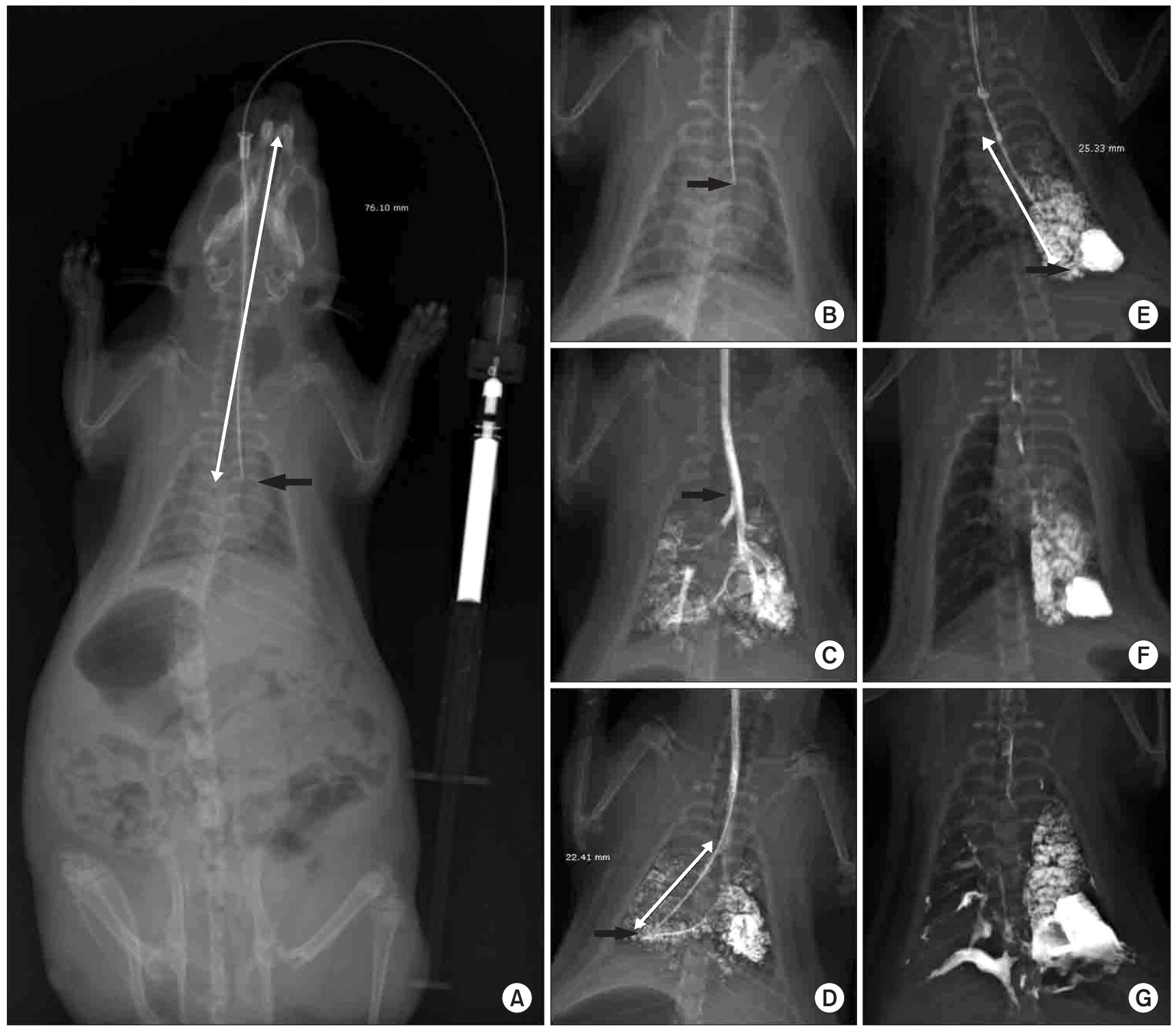

Fig. 5. X-ray findings after catheter insertion and contrast medium administration into the lung. The tip of the epidural catheter was located in the trachea near the carina (arrow in A, B). The distance from the upper incisor to the catheter tip located in the carina was $76.1 \mathrm{~mm}$ (White line in A). The volume of administered contrast medium was $0.3 \mathrm{ml}(\mathrm{C}-\mathrm{F})$ or $0.5 \mathrm{ml}(\mathrm{G})$. The contrast medium dispersed into both lungs because the tip of the catheter was in the trachea (C). The catheter tip was inserted into the lower airway via the left mainstem bronchus using the method described in the text (D). The distance from the carina to the catheter tip was $22.4 \mathrm{~mm}$ (White line in D). The catheter tip was inserted into the lower airway via the right mainstem bronchus using the same method and the contrast medium was injected (E). The contrast medium dispersed into the right lung only because the catheter tip was located in the lower airway distal to the right mainstem bronchus (arrow in E). The distance from the carina to the catheter tip was $25.3 \mathrm{~mm}$ (White line in E). The contrast medium was observed in both lungs after administration. We hypothesized that the large volume of contrast medium administered into the left lung caused it to flow over the carina and into the right lung $(\mathrm{G})$. The double contrast medium was $96 \%$ barium sulfate suspension (SOLOTOP-HD ${ }^{\circledR}$ Seoul, Korea) in $25 \mathrm{~g} / 50 \mathrm{ml}$ PBS. 
This finding may be important for titrating the correct dose or volume of the administered drug for optimal survival rate, which is also likely to be dependent on the toxicity of the compound. In this study, seven rats in group $\mathrm{T}$ died from suspected pulmonary edema immediately after the intratracheal administration of $0.3 \mathrm{ml}$ of bleomycin. However, the rats in group B underwent an intrabronchial administration of the same dose of bleomycin and survived until euthanization 28 days later. Localized gross lesions were observed in their lungs. Gross examination of the lungs from a rat that had died immediately after intratracheal administration of bleomycin revealed pulmonary edema-like lesions throughout the lung (Fig. 3A). Therefore, the requirements for a successful drug administration into a localized area or unilateral lung are as follows: 1) determining the appropriate volume of the drug, 2) maintaining a position with the supine and cephalad end raised 3) the physical characteristics of the drug, and 4) the degree of curvature of the catheter and its correct rotation into the target bronchus. The limitations of this study include the relatively small number of animals and the lack of exact degree and duration of time for raising the cephalad end up and maintaining that position to prevent drug spillage into the opposite lung. This method cannot be used in inhalation studies or in studies related to inflammation caused by catheter insertion. In addition, the experiment's success was based upon gross findings only. Nevertheless, in an experiment with rats, this method offers an advantage over intratracheal administration as it decreases mortality and allows the administration of a drug into a unilateral lung or a localized area without the need for DLTs or more invasive procedures.

\section{References}

1. Matute-Bello G, Frevert CW, Martin TR. Animal models of acute lung injury. Am J Physiol Lung Cell Mol Physiol 2008; 295: L379-99.

2. Moore BB, Hogaboam CM. Murine models of pulmonary fibrosis. Am J Physiol Lung Cell Mol Physiol 2008; 294: L152-60

3. Hirota K, Hashiba E, Kabara S, Yoshioka H, Ishihara H, Matsuki A. Unventilated airway is time-dependently constricted in paralyzed dogs. Anesthesiology 2001; 95: 1480-4.

4. Blanch L, Van der Kloot TE, Youngblood AM, Murias G, Naveira A, Adams AB, et al. Application of tracheal gas insufflation to acute unilateral lung injury in an experimental model. Am J Respir Crit Care Med 2001; 164: 642-7.

5. Stark RA, Nahrwold ML, Cohen PJ. Blind oral tracheal intubation of rats. J Appl Physiol 1981; 51: 1355-6.

6. Almendros I, Gutierrez PT, Closa D, Navajas D, Farre R. One-lung overventilation does not induce inflammation in the normally ventilated contralateral lung. Respir Physiol Neurobiol 2008; 162: 100-2.

7. Vas L. An unusual way to use an endobronchial blocker. Ann Card Anaesth 2001; 4: 40-2.

8. Cohen E. Methods of lung separation. Curr Opin Anaesthesiol 2002; 15: 69-78.

9. Hammer GB, Brodsky JB, Redpath JH, Cannon WB. The Univent tube for single-lung ventilation in paediatric patients. Paediatr Anaesth 1998; 8: 55-7.

10. Uerpairojkit K, Kyokong O. Clinical experiences of one--lung ventilation in adults using the Univent. J Med Assoc Thai 2004; 87(Suppl 2): S190-4.

11. Plopper CG. Structure and function of the lung. In: Monographs on pathology of laboratory animals. Respiratory system, 2nd ed. Edited by Jones TC, Dungworth DL, Mohr U: Springer, Berlin Heidelberg New York Tokyo. 1996, pp 135-50.

12. Dubaybo BA, Thet LA. A technique for unilateral instillation of agents into the rat lung. J Appl Physiol 1985; 59: 266-8. 